STATISTICAL ANALYSIS OF FOURIER COEFFICIENTS

IN A RESTRICTED HARMONIC DIAL

by

\title{
Danny Dyer
}

Department of Mathematics

University of Texas at Arlington

Arlington, Texas 76019

Technical Report No. 24

April, $1975^{\circ}$ 
STATISTICAL ANALYSIS OF FOURIER COEFFICIENTS

IN A RESTRICTED HARMONIC DIAL

\author{
Danny Dyer \\ Department of Mathematics \\ University of Texas at Arlington \\ Arlington, Texas 76019
}

\begin{abstract}
Due to the physical nature of certain periodic data, the harmonic dial points (the Fourier coefficients obtained from harmonic analysis of the data) are sometimes restricted to circular regions in the dialplane. It is proposed that a circular normal distribution (CND) truncated outside a circular region be used to describe the probabilistic behavior of the random phenomena. Recurrence relations for the population moments of a CND truncated outside a circular region are derived. These recurrence relations are used to obtain consistent asymptotically (jointly) normal estimators of the unknown parameters of the distribution. A numerical example based on the harmonic dial points representing the 27day recurrence tendency of the daily international magnetic character figure $c_{i}$ is given to illustrate the theory.
\end{abstract}


INTRODUCTION

The circular normal distribution (CND) is often used to describe the behavion of random phenomena of a geophysical nature. Based on the Mauchly [1940] test, the hamonic dial points (the Fourier coefficients obtained from harmonic analysis of observations of periodic phenomena) representing, for example, (a) the 27-day recurrence tendency of the daily international magnetic character-figure $C_{i}$ [Bartels, 1935], (b) the westerly component of wind in the study of tidal oscillations in the upper atmosphere [Haurwitz, 1964], and $(c)$ the diurnal variation of cosmic ray intensity [Forbush, 1966] may be treated as observations from a CND. However, the "cloud" of points is sometimes restricted to a circular region in the plane of the hammonic dial. For example the limitation of $c_{i}$ to values between 0.0 and 2.0 excludes amplitudes of sine-waves for a 27-day period beyond the theoretical 1 imit of $1.274 C_{i}$ (furnished, for instance, by a succession of 14 days with $C_{i}=2.0$ followed by 13 days with $\left.C_{i}=0.0\right)$. Perhaps then, in this situation, a CND truncated outside a circular region is a more adequate distribution for the dial points. This distribution has been discussed by Dyer [1974] in a study of gunfire pattern restricted to a circular target.

In this paper we establish recurrence relations for the population moments of a CND truncated outside a circular region. These recurrence relations are used to obtain consistent asymptotically (jointly) normal estimators of the unknown parameters of the distribution. The theory is illustrated by a numerical example based on data of the daily international magnetic character-figure $C_{i}$ for 27-day periods. 
RECURRENCE RELATIONS FOR POPULATION MOMENTS

The density function of a two-dimensional random variable $(U, V)$ having a CND truncated outside $C=\left\{(u, v):\left(u-h_{1}\right)^{2}+\left(v-h_{2}\right)^{2} \leq R^{2}\right\}$, where the center $\left(h_{1}, h_{2}\right)$ is known and the radius $R$ may or may not be known, is

$$
f_{U, V}(u, v)=p\left(u, v ; \mu_{1}, \mu_{2}, \sigma^{2}\right) / P \text { for }(u, v) \text { e } C
$$

where

$$
p\left(u, v ; \mu_{1}, \mu_{2}, \sigma^{2}\right)=\left(1 / 2 \pi \sigma^{2}\right) \exp \left\{-\left[\left(u-\mu_{1}\right)^{2}+\left(v-\mu_{2}\right)^{2}\right] / 2 \sigma^{2}\right\}
$$

and

$$
p=\int_{C} \int p\left(u, v ; \mu_{1}, u_{2}, \sigma^{2}\right) d u d v
$$

It will be convenient to translate the origin in the $u v$-plane to $\left(h_{1}, h_{2}\right)$. The density function of $(X, Y)=\left(U-h_{1}, V-h_{2}\right)$ is

$$
f_{X, Y}(x, y)=p\left(x, y ; \xi_{1}, \xi_{2}, \sigma^{2}\right) / P \text { for } 0 \leq x^{2}+y^{2} \leq R^{2} \text {, }
$$

where $\xi_{i}=\mu_{i}-h_{i}, \quad i=1,2$.

Taking the first partial derivative of (2) with respect to $x$ gives

$$
\partial f_{X, Y^{(x, y) / \partial x}=-\left(1 / \sigma^{2}\right)\left(x-\xi_{1}\right) f_{X, Y}(x, y)}
$$

Upon multiplying both sides of (3) by $x^{r} y^{s}$, where $r$ and $\theta$ are nonnegative integers, and integrating over $C$, we have

$$
A_{x}=-\left(1 / \sigma^{2}\right) \mu_{r+1, \theta}^{\prime}+\left(\xi_{1} / \sigma^{2}\right) \mu_{r, 8}^{\prime},
$$


where

$$
\mu_{r, 8}^{\prime}=E\left(X^{r} Y^{B}\right)
$$

and

$$
\begin{aligned}
& A_{x}=\iint_{C} x^{p} y^{B}\left[\partial f_{X, Y}(x, y) / \partial x\right] d x d y \\
& =\int_{-R}^{R}\left(R^{2}-y^{2}\right)^{r / 2} y^{8} f_{X, Y}\left(\left(R^{2}-y^{2}\right)^{1 / 2}, y\right) d y \\
& +(-1)^{r+1} \int_{-R}^{R}\left(R^{2}-y^{2}\right)^{r / 2} y^{\theta} f_{X, Y}\left(-\left(R^{2}-y^{2}\right)^{1 / 2}, y\right) d y \quad-r \mu_{r-1,8}^{\prime} \\
& =(-1)^{8} R^{p+\theta+1} \int_{-\pi}^{\pi} \sin ^{n+1} \theta \cos ^{8} \theta f_{X, Y}(R \sin \theta,-R \cos \theta) d \theta \quad-n \mu_{Y-1, \theta}^{\prime}
\end{aligned}
$$

with the last term on the right-hand side of (5) vanishing when $r=0$. Thus we have the following recurrence relation

$$
\begin{aligned}
& (-1)^{r+1} R^{r+\theta+1} \int_{0}^{2 \pi} \sin ^{r+1} \theta \cos ^{\theta} \theta f_{X, Y}(-R \sin \theta, R \cos \theta) d \theta \\
& =r \mu_{r-1, \theta}^{\prime}-\left(1 / \sigma^{2}\right) \mu_{r+1, \theta}^{\prime}+\left(\xi_{1} / \sigma^{2}\right) \mu_{r, s}^{\prime} \text { for } r \geq 0, \theta \geq 0,
\end{aligned}
$$

where the first term on the right-hand side of (6) vanishes when $r=0$. Using a similar method on the first partial derivative of (2) with respect to $y$, we have 


$$
\begin{aligned}
& (-1)^{\theta+1} R^{r+\theta+1} \int_{0}^{2 \pi} \sin ^{8+1} \theta \cos ^{r} \theta f_{X, Y}(R \cos \theta,-R \sin \theta) d \theta \\
& =8 \mu_{r, 8-1}^{\prime}-\left(1 / \sigma^{2}\right) \mu_{r, \theta+1}^{\prime}+\left(\xi_{2} / \sigma^{2}\right) \mu_{r, \theta}^{\prime} \text { for } r \geq 0, \theta \geq 0,
\end{aligned}
$$

where the first term on the right-hand side of (7) vanishes when $B=0$. The integrals in (6) and (7) may be written in terms of modified Bessel functions [e.g., Gröbner and Hofreiter, 1966, p. 144]; the values of $P$ are tabled in Owen [1962, p. 172].

\section{ESTIMATION OF PARAMETERS}

Case 1. $R$ is known.

The maximum likelihood estimators (MLE) of the unknown parameters $\xi_{1}, \xi_{2}, \sigma^{2}$ are the solution to a system of nonlinear equations. Dyer [1974] has given an iterative technique to solve this system. By using the recurrence relations $(6)$ and $(7)$ and a "modified" method of moments (replace population moments by corresponding sample moments), we shall obtain estimators which are much easier to compute than the MLE. It should be noted that the "usual" method of moments (equate population moments to corresponding sample moments), which uses the lowest-order sample moments possible, leads to the same nonlinear equations as given by the method of maximum likelihood.

Let

$$
\begin{aligned}
& Q_{1}(r, \theta)=\int_{0}^{2 \pi} \sin ^{r+1} \theta \cos ^{\theta} \theta f_{X, Y}(-R \sin \theta, R \cos \theta) d \theta \\
& Q_{2}(r, \theta)=\int_{0}^{2 \pi} \sin ^{\theta+1} \cos ^{r} \theta f_{X, Y}(R \cos \theta,-R \sin \theta) d \theta .
\end{aligned}
$$


Since

$$
\begin{aligned}
& Q_{1}(0,0)-Q_{1}(0,2)-Q_{1}(2,0)=0, \\
& Q_{2}(0,0)-Q_{2}(2,0)-Q_{2}(0,2)=0, \\
& Q_{1}(1,2)+Q_{1}(3,0)-Q_{1}(1,0)+Q_{2}(2,1)+Q_{2}(0,3)-Q_{2}(0,1)=0,
\end{aligned}
$$

we have the following system of equations in matrix form

$$
\begin{aligned}
& {\left[\begin{array}{ccc}
\mu_{2,0}^{\prime}+\mu_{0,2}^{\prime}-R^{2} & 2 \mu_{1,0}^{\prime} & 0 \\
0 & 2 \mu_{0,1}^{\prime} & \mu_{2,0}^{\prime}+\mu_{0,2}^{\prime}-R^{2} \\
\mu_{1,2}^{\prime}+\mu_{3,0}^{\prime}-R^{2} \mu_{1,0}^{\prime} & 2\left(2 \mu_{2,0}^{\prime}+2 \mu_{0,2}^{\prime}-R^{2}\right) & \mu_{2,1}^{\prime}+\mu_{0,3}^{\prime}-R^{2} \mu_{0,1}^{\prime}
\end{array}\right]\left[\begin{array}{c}
\xi_{1} \\
\sigma^{2} \\
\xi_{2}
\end{array}\right]} \\
& =\left[\begin{array}{l}
\mu_{3,0}^{\prime}+\mu_{1,2}^{\prime}-R^{2} \mu_{1,0}^{\prime} \\
\mu_{0,3}^{\prime}+\mu_{2,1}^{\prime}-R^{2} \mu_{0,1}^{\prime} \\
\mu_{4,0}^{\prime}+\mu_{0,4}^{\prime}+2 \mu_{2,2}^{\prime}-R^{2}\left(\mu_{2,0}^{\prime}+\mu_{0,2}^{\prime}\right)
\end{array}\right] .
\end{aligned}
$$

Upon replacing $\mu_{r, \theta}^{\prime}$ in (9) by $m_{r, \theta}^{\prime}=(1 / n) \Sigma x_{i}^{r^{2}} y_{i}^{s}$, where $n$ is the number of paired observations $\left(X_{i}, Y_{i}\right)$, and denoting the estimators of $\xi_{1}, \xi_{2}, \sigma^{2}$ by $\hat{\xi}_{1}, \hat{\xi}_{2}, \hat{\sigma}^{2}$, we have 


$$
\begin{aligned}
& \hat{\xi}_{1}=\frac{a_{4}\left(a_{3} a_{6}-a_{1} a_{5}\right)+a_{2}\left(a_{1} a_{7}-a_{6}{ }^{2}\right)}{a_{1}\left(a_{3} a_{6}-a_{1} a_{5}+a_{2} a_{4}\right)} \\
& \hat{\xi}_{2}=\frac{a_{6}\left(a_{2} a_{4}-a_{1} a_{5}\right)+a_{3}\left(a_{1} a_{7}-a_{4}{ }^{2}\right)}{a_{1}\left(a_{3} a_{6}-a_{1} a_{5}+a_{2} a_{4}\right)} \\
& \hat{\sigma}^{2}=\frac{\left(a_{6}{ }^{2}-a_{1} a_{7}+a_{4}{ }^{2}\right)}{2\left(a_{3} a_{6}-a_{1} a_{5}+a_{2} a_{4}\right)}
\end{aligned}
$$

where

$$
\begin{aligned}
& a_{1}=m_{2,0}^{\prime}+m_{0,2}^{\prime}-R^{2}, a_{2}=m_{1,0}^{\prime}, a_{3}=m_{0,1}^{\prime}, a_{4}=m_{1,2}^{\prime}+m_{3,0}^{\prime}-R^{2} m_{1,0}^{\prime}, \\
& a_{5}=2 m_{2,0}^{\prime}+2 m_{0,2}^{\prime}-R^{2}, a_{6}=m_{2,1}^{\prime}+m_{0,3}^{\prime}-R^{2} m_{0,1}^{\prime}, \\
& a_{7}=m_{4,0}^{\prime}+m_{0,4}^{\prime}+2 m_{2,2}^{\prime}-R^{2}\left(m_{2,0}^{\prime}+m_{0,2}^{\prime}\right) .
\end{aligned}
$$

The estimators given by (10) are consistent asymptotically (jointly) nomal. Consistency follows from the fact that a rational function of sample moments converges in probability to the same rational function of corresponding population moments [Cramér, 1946, p. 358]. Since the estimators are continuous functions of sample moments with continuous first and second order partial derivatives with respect to the sample moments, their asymptotic joint distribution is nomal [Cramér, 1946, p.366]. Finally, it should be noted that when $R \rightarrow \infty$, the estimators (10) are equivalent to the method of moments (and maximum likelihood) estimators of the parameters of an untruncated CND.

Case 2. $R$ is unknown.

When $R$ is unknown, the last equation of the system (8) may be replaced by the two equations

$$
\begin{aligned}
& Q_{1}(1,2)+Q_{1}(3,0)-Q_{1}(1,0)=0 \\
& Q_{2}(2,1)+Q_{2}(0,3)-Q_{2}(0,1)=0 .
\end{aligned}
$$

From the resulting system the following quartic equation in $R^{2}$ can be obtained 


$$
\alpha_{1}\left(R^{2}\right)^{4}+\alpha_{2}\left(R^{2}\right)^{3}+\alpha_{3}\left(R^{2}\right)^{2}+\alpha_{4}\left(R^{2}\right)+\alpha_{5}=0
$$

where

$$
\begin{aligned}
& \alpha_{1}=\beta_{1}-\beta_{1}^{*}, \alpha_{2}=\beta_{2}+\beta_{1} \beta_{4}-\beta_{2}^{*}-\beta_{1}^{*} \beta_{4}^{*}, \\
& \alpha_{3}=\beta_{3}+\beta_{2} \beta_{4}+\beta_{1} \beta_{5}-\beta_{3}^{*}-\beta_{2}^{*} \beta_{4}^{*}-\beta_{1}^{*} \beta_{5}^{*}, \\
& \alpha_{4}=\beta_{3} \beta_{4}+\beta_{2} \beta_{5}-\beta_{3}^{*} \beta_{4}^{*}-\beta_{2}^{*} \beta_{5}^{*}, \quad \alpha_{5}=\beta_{3} \beta_{5}-\beta_{3}^{*} \beta_{5}^{*} ;
\end{aligned}
$$

and

$$
\begin{aligned}
& \beta_{1}=m_{2,0}^{\prime}-m_{1,0}^{\prime 2}, \\
& \beta_{2}=2 m_{1,0}^{\prime}\left(m_{1,2}^{\prime}+m_{3,0}^{\prime}\right)-m_{2,0}^{\prime}\left(m_{2,0}^{\prime}+m_{0,2}^{\prime}\right)-\left(m_{2,2}^{\prime}+m_{4,0}^{\prime}\right), \\
& \beta_{3}=\left(m_{2,2}^{\prime}+m_{4,0}^{\prime}\right)\left(m_{2,0}^{\prime}+m_{0,2}^{\prime}\right)-\left(m_{1,2}^{\prime}+m_{3,0}^{\prime}\right)^{2}, \\
& \beta_{4}=2\left(m_{0,2}^{\prime 2}-m_{2,0}^{\prime}-2 m_{0,2}^{\prime}\right), \\
& \beta_{5}=\left(m_{2,0}^{\prime}+3 m_{0,2}^{\prime}\right)\left(m_{2,0}^{\prime}+m_{0,2}^{\prime}\right)-2 m_{0,1}^{\prime}\left(m_{2,1}^{\prime}+m_{0,3}^{\prime}\right),
\end{aligned}
$$

and $B_{i}^{*}$ is $B_{i}$ in which $m_{r, g}^{\prime}$ is replaced by $m_{\beta, f^{\prime}}^{\prime}$. Upon solving (11) for $R^{2}$ (and discarding all roots which are inadmissible, i.e., negative, imaginary, and those which give a circular region $C$ which excludes an observation in the sample), the positive square root is the estimate of $R$. The estimates of $\xi_{1}, \xi_{2}, \sigma^{2}$ are now given by (10) using the estimate of $R$ found from (11).

\section{APPLICATIONS}

In a classical paper dealing with statistical methods in geophysical periodicities, Bartels [1935] considered the daily international magnetic character-figure, $c_{i}$, for each of the 10,206 days between January 11,1906 
and December 20,1933 (solar rotation numbers 1001 to 1378 , inclusive). For each of the 378 solar rotations, the hamonic coefficients of the sine-wave of frequency one of 27-day periods was computed and graphically displayed in a harmonic dial [see Bartels, 1935]. Mauchly's statistic $L_{e}$ has the value $0.99917 ; \operatorname{Prob}\left(L_{e}\right)=0.732$. Hence the CND hypothes is is accepted.

However, $C_{i}$ is limited by definition to values between 0.0 and 2.0 thus excluding amplitudes of 27-day sine-waves over the theoretical limit of $1.274 C_{i}$ (furnished, for instance, by a succession of 14 days with $C_{i}=2.0$ followed by 13 days with $\left.C_{i}=0.0\right)$. Thus $\operatorname{Prob}\left(u^{2}+V^{2} \leq 1.274^{2}\right)=1$, where $(U, V)$ is a two-dimensional random variable representing the hamonic coefficients for a given 27-day sine-wave $(U$ is positive upward, $V$ is positive towards the right). Furthermore, there is strong evidence of the existence of an $R$ such that $\operatorname{Prob}\left(U^{2}+V^{2} \leq R^{2}\right)=I$, where $R<1.274 C_{i}$. of the 378 dial points, only four have amplitudes in excess of $0.60 C_{i} \mathrm{wit}^{+}$ the maximum amplitude of $0.760 C_{i}$ occurring on solar rotation number 1208 , day $1=$ May 1 , 1921. As a matter of fact, this maximum amplitude has not been exceeded as of solar rotation number 1931 , day $1=$ October 11,1974 . In fact, only one other dial point (solar rotation number 1463 , day $1=$ March 7,1940$)$ has an amplitude beyond $0.70 C_{i}$. Taking the center of the harmonic dial to be the origin in the $u v$-plane, the dial points may be taken as observations from a CND truncated outside the circular region $C=\left\{(u, v): u^{2}+v^{2} \leq R^{2}\right\}$, where $R$ is unknown.

The sample moments are $\left(h_{1}=h_{2}=0\right)$ 


$$
\begin{aligned}
& m_{1,0}^{\prime}=-0.1664497 \times 10^{-1} \quad m_{0,1}^{\prime}=0.3117910 \times 10^{-1} m_{1,1}^{\prime}=-0.1931035 \times 10^{-2} \\
& m_{2,0}^{\prime}=3.5096135 \times 10^{-2} \quad m_{0,2}^{\prime}=3.5702740 \times 10^{-2} \quad m_{2,1}^{\prime}=0.5753302 \times 10^{-3} \\
& m_{3,0}^{\prime}=-0.5871882 \times 10^{-3} \quad m_{0,3}^{\prime}=2.0064549 \times 10^{-3} \quad m_{3,1}^{\prime}=0.0265356 \times 10^{-4} \\
& m_{4,0}^{\prime}=36.8958798 \times 10^{-4} m_{0,4}^{\prime}=36.9420517 \times 10^{-4} \quad m_{1,2}^{\prime}=-0.8169941 \times 10^{-3} \\
& m_{1,3}^{\prime}=-3.4759780 \times 10^{-1} \\
& m_{2,2}^{\prime}=13.3861316 \times 10^{-4} \text {. }
\end{aligned}
$$

From (11),

$$
8848\left(R^{2}\right)^{4}-7921\left(R^{2}\right)^{3}+1703\left(R^{2}\right)^{2}-131\left(R^{2}\right)+3=0
$$

The solutions are $R^{2}=0.62305,0.04002,0.11608 \pm 0.01113 i$. The estimate of $R$ is taken to be

$$
\hat{R}=0.78933 C_{i}
$$

From (10),

$$
\hat{\mu}_{1}=-0.01833 C_{i}, \quad \hat{\mu}_{2}=0.03440 C_{i}, \partial^{2}=0.03459 C_{i} .
$$

Thus the estimated center of mass is $(-0.01833,0.03440)$; the amplitude of the average wave is 0.03898 ; the radius of the probable error circle for single waves is $\sqrt{-2 \ln (1-\hat{B} / 2)} \hat{\sigma}=0.21896(\hat{P}$ is the estimate of $P$ based on $\left.\hat{\mu}_{1}, \hat{\mu}_{2}, \hat{\sigma}^{2}\right)$; and the radius of the probable error circle for the average wave is $\sqrt{-2 \ln (1-B / 2)} \hat{\sigma} / \sqrt{378}=0.01126$ (all in units of $c_{i}$ ). For samples of size 378 from (1) with $h_{1}=h_{2}=0$, we hypothesize $H$ : the average wave 
has zero amplitude, i.e., $\mu_{1}=\mu_{2}=0$. Then the probability an average wave for any sample of size 378 has amplitude $\geq k x_{0}$, where $k$ is a positive constant and $\boldsymbol{r}_{0}$ is the radius of the probable error circle for the hypothesized average wave, is

$$
1-\frac{1-(1-P / 2)^{k^{2}}}{P}
$$

In this example, under the hypothesis $H$, the probability the average wave has amplitude $\geq 0.03898$ is

$$
1-\frac{1-(1-P / 2)^{3.46^{2}}}{\hat{P}}=0.0001
$$

We, therefore, reject the hypothesis $H$ and conclude the average wave is statistically significant [Bartels, 1932]. It should be noted that if an untruncated $C N D$ is used, the indication of statistical significance is much weaker (one-tenth as strong) than the one found above. 


\section{REFERENCES}

Bartels, J., Statistical methods for research on diurnal variations, Terrestrial Magnetiom and Atmosphemic Electricity, 37, 291, 1932. Bartels, J., Random fluctuations, persistence, and quasi-persistence in geophysical and cosmical periodicities, Terrestrial Magnetism and Atmospheric Electriaity, 40, 1, 1935.

Cramer, H., Mathematical Methods of Statistice, Princeton University Press, Princeton, N.J., 1946.

Dyer, D. D., Estimation in a truncated circular nomal distribution with ballistic applications, Operations Research, 22, 197, 1974.

Forbush, S. E., Time-variations of cosmic rays, in Encyctopedia of Phyoics, vol. 49 (part 1), edited by S. Flïgge and J. Bartels, pp. 171-174, Springer-Verlag, New York, 1966.

Gröbner, W. and Hofreiter, N., Integraltafel, Zweiter Teit, Bestimmte Integrate, Springer-Verlag, New York, 1966.

Haurwitz, B., Tidal phenomena in the upper atmosphere, Technical Note No. 58, Wonld Meteorological Organization, 1964.

Mauchly, J.W., A significance-test for ellipticity in the harmonic dial, Terrestrial Magnetiam and Atmoaphemic Eleotricity, 45, 145, 1940. Owen, D. B., Handbook of Statistical Tables. Addison-Wesley, Reading, Mass., 1962. 